\title{
Control comunitario de las infecciones endémicas: pensando los haceres sanitarios colectivos de los campesinos de los Andes peruanos
}

\author{
Community-based control of infectious diseases: \\ reflecting on the collective health practices \\ of Andean Peruvian peasants
}

Julio Alberto Wong-Un 1,2

\footnotetext{
1 Departamento de Endemias Samuel Pessoa, Escola Nacional de Saúde Pública, Fundação Oswaldo Cruz. Rua Leopoldo Bulhões 1480, Rio de Janeiro, $R J$ 21041-210, Brasil. 2 Centro de Estudos e Pesquisas da Leopoldina (CEPEL). Rua Uranos 496, sala 401, Rio de Janeiro, $R J$ 21060-070, Brasil. wongunja@plugue.com.br
}

\begin{abstract}
Discussing four rural experiences with leishmaniasis and malaria control in the Peruvian Andes, this paper analyzes concepts of peripheral health cultures and underlying logic in practical action. Peripheral health cultures in this case refer to the planning, organizing, and intervention abilities used to cope with relevant local health problems. The logic of practical action refers to the constant creation of specific action modes, different from those of other groups. In conclusion, this paper suggests the transformation of official disease control programs by incorporating such forms of logic, characterized by solidarity, inclusion, and cultural appropriateness.

Key words Consumer Participation; Community Participation; Communicable Disease Control; Leishmania; Malaria
\end{abstract}

Resumen A partir del caso de cuatro experiencias andinas peruanas de control de leishmaniasis y malaria, se discuten los conceptos de culturas sanitarias periféricas y lógicas prácticas de la acción. Las culturas sanitarias periféricas se refieren a las habilidades de planificación, organización y acción sobre problemas sanitarios colectivos relevantes realizadas por grupos sociales periféricos. Y las lógicas prácticas de la acción colectiva implican la creación permanente de formas de acción diferenciadas de otros grupos (profesionales, trabajadores de salud formales) que intervienen en la salud local. Se concluye sugiriendo la necesidad de transformar las lógicas de acción de los programas oficiales, incorporando las lógicas inclusivas, solidarias y culturalmente apropiadas generadas por las experiencias rurales de salud realizadas por los propios campesinos.

Palabras clave Participación Comunitaria; Control de Enfermedades Transmisibles; Leishmaniasis; Malaria 


\section{Introducción}

Este artículo aborda la necesidad de mirar con cuidado un conjunto variado y rico de procesos culturales sanitarios que vienen aconteciendo en muchos espacios de América Latina y del Tercer Mundo en general, procesos en los que los usuarios o legos, calificados muchas veces de pasivos o sin cultura (Valla, 1996), elaboran y ejecutan programas o proyectos sociales de control de infecciones endémicas (WongUn, 1997). Esta discusión se inserta en el campo del control comunitario de las enfermedades transmisibles, campo ya definido y trabajado por diversos autores (Al-Mazroa \& Al-Shammari, 1991; Barros et al., 1991; Briceño-León, 1993b; Carneiro \& Antunes, 1994; Dabis, et al., 1989; Delacollette, et al., 1996; Garfield \& Vermund, 1986; Hunter, 1996; Dias \& Dias, 1993; Service, 1993; Vlassof, 1993; Vundule \& Mharakuewa, 1996) aunque todavía poco abordado como punto de partida para propuestas que transformen las prácticas oficiales de control.

Dentro del campo mencionado, concentraré la discusión en la relación entre culturas sanitarias periféricas y control de enfermedades (Briceño-León, 1993a). Para efectos de esta reflexión, las culturas sanitarias son definidas como los haceres colectivos, tácticas y estrategias que las organizaciones comunitarias de salud, pertenecientes a espacios periféricos o subalternos, van construyendo continuamente para afirmar sus prácticas cotidianas de salud (Mata, 1994; Wong-Un, 1997).

A partir de la descripción de cuatro casos peruanos (en los departamentos andinos de Cajamarca y Cuzco), en áreas rurales relativamente aisladas y abandonadas por los servicios públicos de salud, problematizaré las formas de control de las enfermedades endémicas y finalmente situaré la reflexión en el contexto de la crisis de la propuesta sanitaria institucional y señalaré los desafíos para responder a las nuevas configuraciones culturales y espaciales que están apareciendo en las últimas décadas en los espacios periféricos o marginados (Clifford, 1986; Rosaldo, 1989; Sabroza, 1995).

\section{Haceres sanitarios y lógicas prácticas}

Pensar las culturas sanitarias periféricas en América Latina significa pensar los haceres sanitarios y las lógicas de las prácticas de estos grupos. En los últimos treinta años algunas tendencias críticas en la sociología y la antropología (Abu-Lughod, 1991; Appadurai, 1991; Bourdieu, 1974; De Certeau, 1994) han desa- rrollado tentativas para conocer los modos de operación de las prácticas sociales. Sin embargo, en Brasil y Latinoamérica esta reflexión aun no ha sido incorporada completamente ni al quehacer reflexivo de la Salud Pública ni a la construcción de propuestas, tecnologías y prácticas sanitarias. Es, por ello, un tema novedoso para nuestra área (Briceño-León, 1996).

De otro lado, desde el reconocimiento, en los años sesenta, de los mecanismos de opresión que se ejercen finamente, despersonalizados, y de forma microscópica, en las tecnologías institucionales o disciplinares (Foucault, 1979), ha habido diversos intentos por conocer el otro lado de la moneda: es decir, los modos que las personas y grupos construyen para burlar, resistir y recrear esos micro-mecanismos de la opresión (De Certeau, 1994; Scott, 1985). Ello llevó a un interés por caracterizar la práctica, individual y colectiva, como tema de análisis social. En estas tres décadas se han elaborado "esquemas de una teoría de la práctica" (Bourdieu, 1974) y acercamientos a las "tácticas" de las personas y grupos culturales para burlar, en el uso de los bienes simbólicos, las propuestas culturales hegemónicas (De Certeau, 1994). Otros autores, trabajando más en el mundo rural, han caracterizado las "armas de los débiles" (weapons of the weak) como estrategias cotidianas de resistencia - invisibles y disimuladas - practicadas por grupos campesinos (Scott, 1985). Y, finalmente, varios antropólogos contemporáneos inciden en la invención y la creatividad de los marginados, que utilizarían los recursos y ofertas de las culturas oficiales formales para inventar y "tejer" sus subjetividades, identidades y su inserción en el mundo (Appadurai, 1991; Rosaldo, 1989; Starn, 1990). En el uso "crítico" de las ofertas culturales predominantes estarían su rebeldía y su libertad.

Esas obras y reflexiones mostraron que abordar seriamente las prácticas culturales permitiría un "acercamiento teórico (...) construido alrededor de problemas como contradicción, malentendidos, reconocimientos inadecuados, y estrategias de favores, intereses, e improvisaciones, [en contraposición a las] categoría(s) culturales más estáticas y homogeneizantes de reglas, modelos, y textos" (Abu-lughod, 1991:147).

Así, es posible pensar la existencia y operación de "lógicas del hacer colectivo" que no se caracterizarían por ser universos rígidos, cerrados o simplemente normativos, sino que darían espacio a la contradicción, a la comunicación polisignificante, y a la emergencia de la novedad: la invención, la improvisación, y la creación (Appadurai, 1991; Rosaldo, 1989). Los 
haceres colectivos en salud contienen la complejidad del nivel organizacional colectivo, una colectividad participante de varios procesos culturales relacionados a la salud.

Pasando a la especificidad del control comunitario de enfermedades endémicas, podemos afirmar, siguiendo los argumentos anteriores, que en cada proceso emprendido por un grupo comunitario de salud en particular existirían lógicas complejas de acción: lógicas de individuos y lógicas de grupos; modos de priorizar problemas, de percibirlos; de comunicarse con los "beneficiarios"; de planificar y ejecutar las acciones; y de evaluar y replantear su propio trabajo. Y el estilo y el contenido de estas lógicas dependerían, según De Certeau (1994), de la posición del grupo cultural en relación a otros grupos (relaciones de poder, de saber y de hacer). Estas lógicas prácticas incorporarían racionalidades y emotividades, así como la emergencia de la novedad: lo inesperado.

Es importante señalar aquí que estas lógicas sanitarias comunitarias (vividas y construidas grupalmente por individuos diversos, que comparten parcialmente identidades culturales cambiantes en el tiempo y de acuerdo a los contextos, como afirma Clifford, (1986) operan en un contexto de relaciones desiguales de poder y opresión que no solamente son externas, sino que acontecen dentro del mismo grupo cultural periférico (ocurriendo situaciones de explotación entre miembros de la comunidad, e incluso dentro de la familia). Así, no afirmo aquí la pureza cultural o la bondad total de los procesos comunitarios; porque lo comunitario no debe colocarse como camino único, a riesgo de alejarnos de la multiplicidad de acontecimientos contemporáneos y simultáneos caracterizados, justamente, por no ser lineares; y a riesgo de ignorar la necesidad de todas las otras formas de enfrentar los problemas de salud, de acuerdo a niveles de complejidad y configuraciones socio-espaciales. La lógica comunitaria solidaria e inclusiva es uno de los caminos hacia la salud, una forma de emprender procesos sanitarios que incorpora la vivencia de los valores de comunidad, inclusión y solidaridad, dentro del trabajo de salud.

De ello se puede concluir que conocer estos otros haceres sanitarios, distintos de los oficiales, - en el caso del control de enfermedades infecciosas de importancia social - saber cómo operan, cuáles son sus lógicas, y qué valores orientan las direccionalidades, modos de hacer y estilos de trabajo de las personas consideradas legas o ignorantes por el saber sanitario autorizado, permitiría plantear alternativas novedosas a la crisis de los sistemas de salud ofi- ciales (Albuquerque \& Morais, 1996; Osanai, 1994; Dias, 1986). Esta crisis nos coloca ante la necesidad de construir propuestas de intervención a partir de las formas de acción colectiva de salud ya reconocidas y vividas por las culturas locales.

\section{Control de enfermedades endémicas en el mundo rural peruano}

Revisemos algunos aspectos de las culturas sanitarias colectivas de cuatro grupos campesinos dedicados a la salud en los Andes peruanos, específicamente en las regiones de Bambamarca, Chota y Celendín (Cajamarca), en el norte andino, y Ocongate (Cuzco), en el sur andino (Wong-Un, 1997).

En el Perú, los campesinos andinos son triplemente marginados: por vivir en espacios considerados atrasados y aislados; por su condición de pobres; y por ser considerados de raza distinta a la de aquellos que tienen el poder. A pesar de ser el Perú un país eminentemente mestizo, la opresión y el desprecio se expresan también en la presencia incipiente y deformada del Estado en aspectos sociales básicos como salud y educación. Las enfermedades y la muerte son también expresión de la desigualdad que el Perú comparte con todos los países de América Latina.

Con extensas áreas rurales y distantes de los centros de poder económico y político del país, Cajamarca - al norte - y Cuzco - en el sur - son dos de las regiones más pobres del país y en donde la distribución de la riqueza y de la tierra son más injustas. Dentro de estos espacios, las condiciones de vida de las comunidades campesinas - reflejadas parcialmente en los indicadores sociales y constatadas fácilmente al vivir o trabajar en las zonas rurales contrastan con el bienestar de los hacendados, empresarios mineros, comerciantes y empresarios urbanos y productores de leche y carne. Esta situación de acceso desigual se repite en las condiciones de salud de los grupos campesinos y en su precaria relación con los servicios y trabajadores de salud.

La salud ha sido tema frecuente de los esfuerzos de la Sociedad Civil para revertir o disminuir la desigualdad y el sufrimiento de los grupos más pobres en el campo cajamarquino y cusqueño. Tanto la Pastoral Social de la Iglesia, como las Organizaciones no Gubernamentales (ONGs), la cooperación internacional en salud y también el Estado Peruano han impulsado programas rurales de atención básica de salud, de control de enfermedades, de educa- 
ción y de promoción de la salud. Sin embargo, en general, estos proyectos han sido insuficientes en cobertura y permanencia en el tiempo; y muchos de ellos no fueron pensados ni adaptados a las realidades culturales locales (no sólo en términos de propuestas técnico/médicas sino sobre todo en sus propuestas organizativas y de planificación) por lo que se pueden considerar "fracasos", en relación a su legitimación local e impacto social sobre los grupos campesinos, reforzando una situación de marginalidad y de resistencia a las intervenciones externas en el campo de la salud.

Esta situación de "marginalidad sanitaria" ha sido enfrentada históricamente por las medicinas populares y los agentes tradicionales de salud (curanderos, brujos, parteras, herbolarios). Pero, siguiendo la lógica de la creatividad en el uso (Scott, 1985; De Certeau, 1994; Starn, 1990), algunos grupos campesinos han ido incorporando en sus habilidades y prácticas las tecnologías y discursos propuestos por la salud pública, la epidemiología de campo, los programas de control de enfermedades infecciosas, la medicina y epidemiología sociales, y los organismos oficiales de salud.

Lo que se evidencia aquí es que los campesinos agentes de salud comunitaria incorporaron, adaptándolas y transformándolas creativamente, las propuestas y tecnologías con las que convivieron, y de las que fueron inicialmente "objetos" de intervención. A lo largo de los últimos treinta años, cuando, siguiendo las orientaciones de las universidades y escuelas norteamericanas (y las políticas de control social propuestas por el gobierno americano), el campo fue "invadido" por diversas propuestas de desarrollo y bienestar social en salud, y los líderes campesinos (los antiguos y los jóvenes) fueron aprendiendo a utilizar los discursos y las formas de actuar en salud de los "agentes externos”, técnicos o profesionales de salud. Este aprendizaje tuvo resultados diversos: desde experiencias de aumento de control social sobre los grupos subalternos a experiencias transformadoras que expresan valores comunitarios y de soporte social solidario. Dentro de esta última línea, las organizaciones campesinas de salud en Cajamarca y Cuzco - desde sus historias locales y biografías grupales - crearon formas nuevas de controlar algunas de las enfermedades infecciosas endémicas más importantes en sus regiones.

\section{Las organizaciones de promotores de salud de Bambamarca, Celendín y Chota, en Cajamarca}

Por más de veinte años las asociaciones de Agentes Comunitarios de Salud (Promotores de Salud) en Cajamarca realizan un trabajo de promoción, educación y organización campesina, previniendo y controlando diversos problemas. Estas organizaciones de salud comunitaria han ido generando con su práctica diversas tecnologías organizativas, de relación con sus vecinos/usuarios, y formas colectivas de prevenir y controlar las enfermedades más frecuentes.

Entre 1962 y 1992 los campesinos de esta región andina recibieron fuerte influencia de las corrientes progresistas de la Iglesia Católica; y, en los últimos quince años de este periodo, del movimiento campesino de autogobierno y justicia popular llamado Ronda Campesina, dos influencias que imprimieron un carácter ético de "compromiso" y de "misión de vida" a la labor sanitaria de los campesinos. Y aun hoy, después de la desactivación de las corrientes eclesiales progresistas y de la micro fragmentación de las rondas campesinas, los líderes de salud encarnan con su práctica paciente y solidaria el espíritu ético y humanista que aprendieron (y, principalmente, construyeron) a lo largo de los años.

En este sentido, sus acciones colectivas de salud - la planificación de base, las dinámicas organizativas, las campañas de prevención, control e intervención sobre el medio ambiente, y sus acciones de educación y capacitación - pueden entenderse también como parte de procesos de democracia local, lucha social, respeto mutuo y compromiso con el bienestar general. El signo distintivo del trabajo sanitario campesino en Cajamarca es el componente ético y de vivencia de comunidad.

De manera diversa, este autor acompañó a estas organizaciones en el periodo que va de 1990 a 1994, constatando la regularidad de sus acciones, su creatividad, su capacidad para dialogar sobre y cuestionar las propuestas de otros agentes sanitarios locales y regionales (profesionales, trabajadores de salud, dueños de farmacias, autoridades del gobierno). Durante los últimos años las organizaciones comunitarias de salud han tenido que adecuarse a las nuevas condiciones de escaso apoyo externo (Mburu, 1994), con un proceso paciente de articulación regional de todos los Promotores de Salud del Norte Andino peruano. Esto ha permitido aumentar las capacidades y saberes de manera colectiva (intercambio de experien- 
cias y habilidades con grupos de otras provincias o departamentos).

En Bambamarca, capital de la provincia de Hualgayoc, a 100 kilómetros al norte de la ciudad de Cajamarca (capital del departamento), la Asociación de Promotores de Salud contaba hasta 1994 con 90 miembros, de más o menos 70 comunidades campesinas. La mayor parte eran hombres entre 25 y 40 años, líderes que dividían su trabajo de salud con la agricultura y la artesanía de sombreros; y algunos de ellos con cargos locales o distritales de autoridad (Presidente de Rondas, Teniente-Gobernador, Maestro de Escuela, Pastor Evangélico, etc.). La dinámica de organización consistía en: dos grandes reuniones anuales (en enero y agosto) para planificar y evaluar el trabajo decidido por el colectivo; reuniones dominicales en un pequeño local alquilado en el pueblo de Bambamarca, donde se vendían medicamentos, miel y plantas medicinales a los miembros de la organización; y visitas permanentes a las Zonas distantes. Eran los miembros de la Junta Directiva, formada por siete personas que viajaban por todo el distrito, caminando durante varios días para visitar, capacitar, apoyar y supervisar el trabajo de las “zonas”. Cada zona tenía un aproximado de ocho a doce comunidades, distantes entre sí. Se organizaban cursos zonales de dos a tres días, que eran dados por la Junta Directiva, formada por los líderes con más experiencia y conocimientos de salud.

En las asambleas anuales se decidía asumir un tema clave, o problema a ser resuelto, por año. Cada año se incidía en un tema distinto, relacionado al ambiente, a la desnutrición, diarrea, cólera, o a las relaciones con los servicios de salud del Estado. Es en ese proceso de articulación y decisión colectiva que se manifestaba, en mi opinión, la gran diferencia con la verticalidad de las instituciones formales (sean del Estado, privadas o incluso las alternativas, como la Pastoral Social o las Organizaciones No Gubernamentales). El ejercicio de la ciudadanía entre los campesinos se expresa también mediante el eje aglutinador de la salud.

Todos los espacios de decisión eran colectivos y con estímulo permanente de la participación de todos/todas. Durante el periodo que acompañé y visité Bambamarca (1990/1994), este grupo de campesinos estaba saliendo de un proceso de aprendizaje y de reinterpretación de las propuestas de transformación social (de la Iglesia Católica progresista y los Partidos Políticos de izquierda); y de salud comunitaria (Atención Primaria de Salud, Educación y Comunicación Popular en Salud, Pastoral de Salud) para, por razones que no abordaré aquí, pasar a la autogestión de su vida colectiva como agentes de salud.

Esta necesidad nueva de independencia afectó la vida de las organizaciones de salud en por lo menos dos niveles: primero, los promotores fueron llevados a una crisis de orfandad y falta de apoyo externo que produjo la pérdida de varios líderes valiosos que, o desistieron, o pasaron a realizar otras actividades de liderazgo local; y, segundo, obligaron al desarrollo creativo de formas de democracia interna y formas de trabajar la salud (técnicas y discursos sanitarios) que expresan necesariamente "modernidades alternativas" (Appadurai, 1991) por sus formas de practicar la salud comunitaria, con y para la comunidad, como parte de la comunidad e incluyendo más que excluyendo las propuestas de "saber hacer" en salud, profesionales y populares.

Este camino campesino de búsqueda de la autogestión y de construcción de prácticas culturales sanitarias colectivas alternativas, configurando una determinada cultura sanitaria, distaba mucho de ser ideal o de funcionamiento perfecto. Ello porque los campesinos participan de circuitos culturales alternativos/periféricos, y se sitúan (voluntaria o involuntariamente) a contracorriente de las propuestas oficiales o hegemónicas, sea en lo sanitario o en lo gubernamental. Y, por otro lado, porque, como afirma McKnight (1987), el espacio comunitario acepta la imperfección y va generando los espacios donde las personas puedan insertarse, con sus habilidades y deficiencias, al contrario de la esfera de lo institucional, caracterizada por la rigidez y la poca capacidad de tolerar lo imprevisto, lo nuevo o la contradicción.

En Celendín, a unos cien kilómetros de Bambamarca y Cajamarca, la historia de la organización campesina de salud está mucho más relacionada al trabajo sostenido de un pequeño grupo de agentes de pastoral que tomaron el trabajo de salud como tema eje de su trabajo evangelizador y de promoción del desarrollo local. La Asociación de Promotores de Salud recibe, por un lado, apoyo permanente del equipo pastoral local con asesoría profesional, recursos suficientes para un funcionamiento mínimo y capacitación frecuente, en Celendín y fuera de Cajamarca. Pero, por otro lado, los niveles de autonomía son menores, las formas de "hacer' la salud colectiva son más convencionales. Tal vez lo más creativo y revelador es la relación con los pacientes, las formas "invisibles” y "microscópicas”, altamente eficientes, de captar y acompañar a los enfermos.

Celendín viene abordando problemas como malaria por $P$. vivax, endémica en la parte 
selvática de la provincia (y con algunos casos recientes por $P$. falciparum), y leishmaniasis en las dos formas existentes en el Perú: la mucocutánea o espundia y la cutánea, llamada uta en los Andes. En la organización de este control comunitario de enfermedades en Celendín intervinieron varios grupos profesionales de Cajamarca: el proyecto APRISABAC, de la Cooperación Holandesa en Salud, el equipo pastoral de Celendín (con una médica y una religiosa enfermera) e instituciones nacionales de asesoría en pastoral de salud (Servicios Educativos Rurales, SER; el Departamento de Pastoral de Salud, DEPAS; y Cáritas del Perú).

Sin embargo la acción directa y la supervisión de las acciones de prevención y control fueron realizadas por los Promotores de Salud junto con la médica del Equipo Pastoral. Por ejemplo, en el caso de leishmaniasis, se organizó una campaña de captación activa de casos, con visitas en todas las comunidades cubiertas por la organización de promotores de salud. En estas visitas se registraban en cuadernos simples los datos de posibles enfermos (sospechosos) con lesiones cutáneas compatibles con leishmaniasis; previamente, los promotores fueron entrenados en el reconocimiento de heridas. Luego de un periodo que variaba de dos a seis semanas, la médica del equipo pastoral visitaba algunas comunidades donde los promotores la llevaban a los posibles enfermos para la confirmación clínica del diagnóstico y el inicio del tratamiento.

Los promotores, andarillos incansables, visitaban regularmente a los pacientes para ver su progreso en el proceso de cura y también para - dentro de la lógica andina y cristiana popular de una salud ampliada que incorpora activamente las relaciones interpersonales - animarlos, dándoles apoyo y cariño de manera sistemática, encuadrándose dentro de lo que se ha llamado apoyo social o social support (Valla, 1998).

Con la endemia de malaria, la acción de apoyo era semejante. Cuando los promotores conseguían detectar algún enfermo en sus comunidades, además de garantizar el tratamiento farmacológico, garantizaban el apoyo a la persona enferma, la acompañaban en sus idas a los servicios de salud y a veces hasta apoyaban a la familia del paciente.

En Chota, provincia al norte de Hualgayoc, a unos 150 kilómetros de la ciudad de Cajamarca, las acciones contra leishmaniasis resultaron tan eficaces en términos de generación de corrientes de opinión y lobby sanitario que, en pocos años (el trabajo se inició en 1992), el gobierno peruano abrió un servicio especializado en el hospital local (en 1994) para el tratamiento de los casos que antes eran considerados inexistentes por las autoridades sanitarias locales.

A inicios de los años noventa, en Chota, se afirmaba que leishmaniasis no era un problema importante. Esta afirmación es un buen ejemplo de la ceguera profesional o burocrática: lo real es sólo aquello que puedo o quiero ver (Valla, 1996). Los campesinos, participantes como Promotores de Salud de la Pastoral Social del Obispado de Chota (de tendencia más conservadora que sus vecinos de Celendín y Bambamarca), comenzaron a ver rechazados a los enfermos; y éstos comenzaban a desanimar de curarse de una enfermedad lenta, indolora y que, en sus fases iniciales, no alteraba su capacidad de trabajo. Era necesario, pues, un trabajo de estímulo de la acción sanitaria sobre este problema silencioso, silenciado, lento e incontrolado.

Los agentes de salud campesinos pidieron apoyo a la Pastoral Social para capacitarse en la detección y tratamiento de leishmaniasis cutánea. El padre responsable solicitó apoyo del SER y de Cáritas, instituciones ya mencionadas, que iniciaron un conjunto de reuniones con los casi doscientos campesinos y campesinas interesados en saber más sobre esa enfermedad no reconocida por las autoridades sanitarias locales. Poco tiempo después, los diagnósticos rurales hechos por los propios campesinos mostraron un cuadro epidemiológico totalmente distinto a aquel afirmado por los directores locales de salud. La Iglesia entregó regularmente por dos años copia de estos diagnósticos al hospital de Chota. Y, luego de casi tres años, se inauguró el consultorio especializado en leishmaniasis que, por un lado, representa el reconocimiento de una presión popular realizada desde la práctica en salud; y, por otro, representó, lamentablemente, la desactivación del trabajo directo de salud comunitaria con leishmaniasis de la organización de agentes de salud que pasó a resolver otros problemas, aunque sin dejar de ejercer control público sobre el hospital.

\section{La asociación de enfermos de leishmaniasis de Ocongate: la experiencia en un espacio regional andino}

Un caso especial en la historia de los movimientos sociales en salud en el Perú es el caso de los campesinos quechuas del distrito de Ocongate, provincia de Quispicanchis, a unos 
100 kilómetros al sur de la ciudad del Cuzco. Existen en esas comunidades quechuas gran número de enfermos de leishmaniasis que adquirieron la enfermedad en viajes temporales a la parte amazónica del departamento (Quillabamba, La Convención, Huaypetu), a Madre de Dios, o al Brasil, como parte de las estrategias de los campesinos pobres de las zonas altas del Cuzco para contrarrestar la pobreza, consecuencia, entre otros factores, de la baja producción de sus terrenos de cultivo.

La Parroquia de Ocongate viene apoyando a estos enfermos por más de quince años, consiguiendo tratamiento con Glucantime (antimonial pentavalente de origen francés, comercializado en el Perú), diagnóstico y control especializado, y generando alternativas de trabajo. Apoyó también la formación de la Asociación de Enfermos de Leishmaniasis (AELO), que trabaja en la mayoría de comunidades del distrito en la captación, tratamiento y rehabilitación de enfermos. Asimismo, AELO estimuló, progresivamente, la formación de organizaciones similares en otras regiones del Sur Andino.

En el Sur Andino, la espundia, llamada uta en esta región (identificada lingüísticamente con la forma cutánea), es motivo de marginación social por la deformación causada por las lesiones avanzadas y, principalmente, porque el enfermo grave pierde su capacidad de trabajar en el campo y, por tanto, su capacidad de aporte económico y social a la unidad familiar y comunal. Por ello, enfermarse de leishmaniasis es un estigma social, y los enfermos, en general, tienen mayor interés en cuidar de sí mismos. No ocurre lo mismo en el Norte Andino, donde el tipo de enfermedad, con lesiones menores, minimiza su impacto social y cultural.

Entre Cuzco y Puno, el Sur Andino, se concentran más del 50\% de los casos nacionales de leishmaniasis mucocutánea, constituyendo un problema social y de salud pública importante. Tal es la cantidad de casos acumulados que, a finales de la década de los ochenta, se comenzó a gestar un Comité Regional de Lucha contra la Leishmaniasis, en el cual el Estado participaba como parte de un grupo interinstitucional de concertación con presencia de la Iglesia Católica, de ONGs como el Centro de Investigación y Promoción Amazónica (CIPA), y de las Universidades (UNSAAC y UPCH), y grupos de derechos humanos.

Para el trabajo de este comité fueron decisivas la existencia y la fuerza de una organización como la Asociación de Enfermos de Leishmaniasis de Ocongate, AELO, que, mediante una labor de lobby social, consiguió interesar a los varios sectores sociales implicados en el control de la enfermedad.

Los miembros de las asociaciones de enfermos son campesinos del distrito de Ocongate, del resto de la provincia, y de lo que en el Perú se conoce como Provincias Altas del Cuzco. Luego de integrarse a la asociación los enfermos recibían atención médica, tratamiento y control de la evolución de su caso; participaban de cursos y reuniones de capacitación y de las reuniones distritales en la parroquia de Ocongate. De este modo, luego de un tiempo, varios de ellos se formaron como líderes comunitarios de la asociación, pasando a responsabilizarse de varias de las acciones iniciadas por el equipo pastoral, la ONG CIPA, y otros grupos de apoyo al desarrollo comunitario (una situación semejante describen Dedet et al., 1995, en Bolivia).

En un periodo posterior de organización, AELO comenzó a trabajar alternativas de ingreso y empleo para atacar el problema de pobreza que "expulsa" a muchos campesinos hacia las áreas endémicas. La Iglesia local comenzó a trabajar en la Pastoral de Migraciones, para visitar las áreas endémicas, mejorar las condiciones de trabajo de los campesinos en la selva y rescatar a los menores de edad que viajaban y eran explotados en los lavaderos de oro.

\section{Participación y prácticas de salud en las organizaciones rurales comunitarias}

El concepto de culturas sanitarias periféricas esbozado en la primera parte de este artículo se refiere básicamente a las habilidades culturalmente construidas por un grupo definido de agentes sociales en sus acciones de salud colectiva. El concepto se restringe, para nuestra discusión, a las acciones colectivas de salud, ya que la comprensión de las relaciones curador/enfermo o profesional/lego exigiría otros abordajes de la realidad social de los grupos estudiados.

Las formas de hacer o las "lógicas de la práctica” social representan un desplazamiento del ángulo de análisis de "lo social", como ya fue evidenciado por los autores mencionados, del campo estructural al campo "móvil” y "frágil" de las acciones o agency (Giddens, 1979). La contradicción y la emergencia de la novedad sugieren que estas dinámicas de estructura/acción son complejas, y que soluciones simples, lineares, verticales, jerárquicas y unificadoras en el campo del control de enfermedades transmisibles o de grandes endemias no son las más adecuadas para conseguir resulta- 
dos de bienestar sostenido en el tiempo que se incorporen al cotidiano de las personas: a sus “agendas" de bienestar. La constatación de la existencia de lógicas prácticas diferenciadas por grupos culturales, también dentro del mundo de la "salud pública vivida", sugiere que las personas siempre están evaluando críticamente las ofertas que vienen desde los niveles sanitarios formales. Rehaciendo (y a veces invirtiendo radicalmente los objetivos oficiales), estas propuestas y tecnologías inventan formas de actuar la salud colectiva en sus espacios culturales que son formas correspondientes a sus intereses y que pocas veces son entendidas claramente, por el complejo afirmación/negación o conformismo/resistencia o pasividad/rebeldía que usualmente contienen. Estas formas de hacer salud de los grupos periféricos rurales proponen diferencias importantes que deberían transformar la formalidad del control de infecciones endémicas (Dias, 1994; Dias \& Dias, 1993). Pero estos cambios en los programas nacionales de control y en las lógicas prácticas de los planificadores y autoridades de salud no se dan simplemente en la esfera de la "reforma" de los programas.

Tal vez la comprensión racional de las culturas sanitarias periféricas no sea suficiente, demandando nuevos abordajes vivenciales que incorporen experiencias de transformación del conocimiento profesional, cambios profundos en la vida del actor social "autorizado" por la sociedad a "hablar" sobre medicina y salud. Otros caminos que son simples en su posibilidad, pero que representarían quiebras o rupturas radicales en nuestra forma de "democracia interna epistemológica” en relación a los Otros que hasta ahora consideramos poco autorizados, poco cultos, y repletos de prejuicios o “sentido común” y saberes "errados". La transformación requiere vivencia ética, respeto por la diversidad y trabajar permanentemente escuchando, comprendiendo y proponiendo con los "legos" que, ahora sabemos, tienen prácticas sociales en salud que son proyectos distintos de modernidad y sociedad, solidarios e inclusivos.

En los cuatro casos descritos brevemente aquí, los campesinos indígenas o mestizos, de comunidades andinas "atrasadas", hostilizadas y probablemente con procesos de transformación intensos en sus lógicas culturales y económicas, crearon, como ha sido reportado en otras partes, formas propias e inclusivas de prevenir y controlar enfermedades infecciosas endémicas allí donde el mundo formal sanitario estaba ausente o había evidenciado insuficiencias serias, como Briceño-León (1993b) y
Dias \& Dias (1993) refieren. Estas formas culturalmente apropiadas, periféricamente construidas, expresan bien aquel tipo de participación comunitaria en la cual la población es la que realiza las acciones, desde el diseño a la evaluación, convirtiéndose en una mezcla de proveedor de servicios/usuario, mezcla creativa que lleva a la invención de tecnologías y saberes distintos a los usuales en el control de infecciones endémicas (Briceño-León, 1993b).

En los cuatro casos descritos, la presencia fuerte de una ética solidaria y de dinámicas organizativas democráticas, y de elementos de mística y compromiso de trabajo; así como la forma de relación con la población y los enfermos, acompañándolos y siendo parte de sus mundos sociales, son algunas de las diferencias importantes con las propuestas de los programas formales de control de endemias, caracterizados por su verticalidad y por sus estrategias militares de "penetración”, “campaña” y “erradicación” (Dias \& Dias, 1993).

El hecho que los indicadores de morbimortalidad no varíen en muchas de las experiencias comunitarias no debería llevarnos a desecharlas o condenarlas por inútiles. Como Bastien (1992) afirma, el valor central de los líderes comunitarios de salud es social y cultural: ellos permiten articulaciones entre servicios de salud y población; estimulan la participación de las personas; son líderes "reguladores" y no "jerárquicos"; representan bien los intereses de la comunidad; y amplían nuestras concepciones de salud, incluyendo el bienestar social, emocional y económico. Las experiencias de Promotores de Salud en América del Sur siempre han sido poco apoyadas, considerándolas periféricas y poco importantes; y los factores que influyen en la alteración significativa de algunos indicadores de morbilidad sobrepasan la responsabilidad y posibilidad de los agentes comunitarios de salud. Su valor, irremplazable como dice Bastien, está más bien en la construcción de culturas etno-sanitarias que acercan mundos separados y a veces contrapuestos como el formal de los servicios y las comunidades rurales andinas. Y en la creación permanente de culturas sanitarias, fuertemente éticas, fuertemente solidarias, fuertemente emocionales.

Las experiencias de creación e invención de culturas sanitarias por grupos periféricos o de culturas "no-occidentales", que acontecen en varias partes del globo, constituyen alternativas ricas y variadas para reformular y transformar programas oficiales de control de endemias importantes como malaria y leishmaniasis, ya que proponen modos inclusivos y soli- 
darios de relación con las personas, aquel "factor olvidado" en el control de enfermedades infecciosas (Gillett, 1985), y permiten procesos sostenidos en el tiempo y apropiados por las comunidades participantes. Se podría pasar así de un modelo externo a uno de construcción interna; de una forma militar a una forma democrática de enfrentar las enfermedades. Y se daría oportunidad a los profesionales de aprender formas menos disecadoras y mecanicistas de entender la salud.

\section{Agradecimientos}

El autor desea agradecer a las Asociaciones de Promotores de Salud del Norte Andino peruano y de Ocongate, a los equipos pastorales en Cajamarca y Cuzco; y a los profesionales que los apoyan permanentemente.

\section{Referencias}

ABU-LUGHOD, L., 1991. Writing against culture. In: Recapturing Anthropology (R. Fox, ed.), pp.137162, Santa Fe: School of American Research Press.

ALBUQUERQUE, M. F. M. \& MORAIS, H. M., 1996. Descentralización del control de las endemias: modelo de intervención para combatir la filariasis de Bancroft. Boletín de la Oficina Sanitaria Panamericana, 121:75-86.

AL-MAZROA, Y. \& AL-SHAMMARI, S., 1991. Community participation and attitudes of decision-makers towards community involvement in health development in Saudi Arabia. Bulletin of the World Health Organization, 69: 43-50.

APPADURAI, A. 1991. Global ethnoscapes. Notes and queries for a transnational anthropology. In: $R e-$ capturing Anthropology (R. Fox, ed.), pp. 191-210, Santa Fe: School of American Research Press.

BARROS, F. C.; VICTORA, C. G.; FORSBERG, B.; MARANHÃO, A. G.; STEGEMAN, M.; GONZALEZRICHMOND, A.; MARTINS, R.M.; NEUMAN, Z.A.; MCAULIFFE, J. \& BRANCO Jr., J.A., 1991. Management of childhood diarrhea at the household level: a population survey in north-east Brazil. Bulletin of the World Health Organization, 69: 59-65.

BASTIEN, J., 1992. Drum and Sthetoscope. Salt Lake City: University of Utah Press.

BOURDIEU, P., 1974. Outline of a Theory of Practice. Cambridge: Cambridge University Press.

BRICEÑO-LEON, R., 1993a. La enfermedad de Chagas: una Construción social. In: Las Enfermedades Tropicales en la Sociedad Contemporánea ( $\mathrm{R}$. Briceño-León \& J. C. P. Dias, org.), pp. 257-282, Caracas: FERV.
BRICEÑO-LEON, R., 1993b. Venezuela: retos para alcanzar la participación comunitaria en el control de las enfermedades tropicales. Fermentum, 4: 165-176.

BRICEÑO-LEON, R., 1996. Siete tesis sobre la educación sanitaria para la participación comunitaria. Cadernos de Saúde Pública, 12: 7-30.

CARNEIRO, M. \& ANTUNES, C., 1994. Avaliação de eficácia do Programa de controle da doença de Chagas: aspectos metodológicos. Cadernos de Saúde Pública, 10 (Sup. 2): 261-272.

CLIFFORD, J., 1986. Parcial truths: introduction. In: On Writing Culture: the Poetics and Politics of Ethnography (J. Clifford \& G. Marcus, ed.), pp.126, Berkeley: University of California Press.

DABIS F.; BREMAN J.G.; ROISIN A.J. \& HABA F., 1989. Monitoring selective components of primary health care: methodology and community assessment of vaccination, diarrhoea, and malaria practices in Conakry, Guinea. Bulletin of the World Health Organization, 67: 675-684.

DE CERTEAU, M., 1994. A Invenção do Cotidiano. Petrópolis: Vozes.

DEDET, J. P.; MELOGNO, R. \& CARDENAS, F., 1995. Rural campaign to diagnose and treat mucocutaneous leishmaniasis in Bolivia. Bulletin of the World Health Organization, 73: 339-345.

DELACOLLETTE, C.; VAN DER STUYFT, P. \& MOLIMA, K., 1996. Using community health workers for malaria control: experience in Zaire. Bulletin of the World Health Organization, 74: 423-430.

DIAS, J. C. P., 1994. Ecological aspects of the vectorial control of Chagas disease in Brazil. Cadernos de 
Saúde Pública, 10 (Sup. 2): 352-358.

DIAS, J.C. P, 1986. Participação comunitária em programas de saúde. Revista Brasileira de Malariologia e Doenças Tropicais, 38:103-110.

DIAS, J. C. P. \& DIAS, R. B., 1993. La necesidad de investigación social y económica para las acciones de control de las enfermedades tropicales. In: Las Enfermedades Tropicales en la Sociedad Contemporánea (R. Briceño-León \& J. C. P. Dias, org.), pp.29-50, Caracas: FERV.

FOUCAUlT, M., 1979. A Microfísica do Poder. São Paulo: Graal.

GARFIELD, \&.; VERMUND, S. 1986. Health education and community participation in mass drug administration for malaria in Nicaragua. Social Science and Medicine, 22: 869-877.

GIDDENS, A., 1979. Central problems in social theory: Action, structure and contradiction in social analysis. Berkeley \& Los Angeles: University of California Press.

GILLETT, J. D., 1985. The behavior of Homo sapiens, the forgotten factor in the transmission of tropical disease. Transactions of the Royal Society of Tropical Medicine and Hygiene, 79:12-20.

HUNTER, J., 1996. An introduction to guinea worm on the eve of its departure: dracunculiasis transmission, health effects, ecology and control. Social Science and Medicine, 43:1399-1425.

MATA, J. A. I, 1994. A Epidemia de Cólera no Peru Como um Evento Social. As Representações das Lideranças Comunitárias de Villa El Salvador. Tese de Mestrado, Rio de Janeiro: Escola Nacional de Saúde Pública/Fundação Oswaldo Cruz.

MBURU, F. M., 1994. Whiter community health workers in the age of structural adjustment? Social Science and Medicine, 39: 883-885.

MCKNIGHT, J., 1987. Regenerating community. Social Policy, Winter:54-58.

OSANAI, C. 1994. Descentralização do controle de endemias: processos/produtos; controle popular. In: Descentralização do Controle de Endemias (Ministério da Saúde, Fundação Nacional de Saú- de, orgs), pp.42-46, Brasília: Fundação Nacional de Saúde, Ministério da Saúde, Departamento de Operações. Coordenação de Controle de Doenças Transmitidas por Vetores.

ROSALDO, R., 1989. Culture and Truth. The Remaking of Social Analysis. Boston: Beacon Press.

SABROZA, P., 1995. Doenças transmissíveis: ainda um desafio. In: Os Muitos Brasis. Saúde e População nos 80 (M. C. S. Minayo, org.), pp. 177-244, Rio de Janeiro, São Paulo: Hucitec/Rio de Janeiro: Abrasco.

SCOTT, J., 1985. Weapons of the Weak. Everyday Forms of Peasant Resistance. New Haven: Yale University Press.

SERVICE, M W., 1993. Community participation in vector-borne disease control. Annals of Tropical Medicine and Pararasitology, 87: 223-234.

STARN, O., 1990. Con los Llanques todo Barro. Reflexiones sobre Rondas Campesinas, Protesta Rural y Nuevos Movimientos Sociales. Lima: IEP.

VALLA, V., 1996. A crise da interpretação é nossa: procurando compreender a fala das classes subalternas. Educação e Realidade, 21:177-190.

VALLA, V., 1998. Apoio social e saúde: buscando compreender a fala das classes populares. In: $E d u$ cação Popular Hoje (M. V. Costa, org.), São Paulo: Ed. Loyola (no prelo).

VLASSOF, C., 1993. Estado actual de la investigación social y económica sobre las enfermedades tropicales. In: Las Enfermedades Tropicales en la Sociedad Contemporánea (R. Briceño-León \& J. P. Dias, orgs.), pp.15-28, Caracas: FERV.

VUNDULE, C \& MHARAKUEWA, S., 1996. Knowledge, practices and perceptions about malaria in rural communities of Zimbabwe: relevance to malaria control. Bulletin of the World Health Organization, 74: 55-60.

WONG-UN, J. A., 1997. Valores Comunitarios y Control de Endemias Transmisibles: Cultura, Control Social y Salud en los Campesinos del Norte Andino Peruano. Dissertação de Mestrado, Rio de Janeiro: Escola Nacional de Saúde Pública, Fundação Oswaldo Cruz. 